\title{
Cysteine Cathepsins and Matrix Metalloproteases Among Breast Cancer Patients
}

\author{
Solomon Tsegaye' \\ Mohammed Mehdi iD ${ }^{2}$ \\ Wajana L Labisso (iD ${ }^{3}$ \\ Daniel Seifu Melka ${ }^{2}$ \\ 'Department of Biochemistry, College of \\ Health Sciences, Assela University, Addis \\ Ababa, Ethiopia; ${ }^{2}$ Department of \\ Biochemistry, College of Health Sciences, \\ Addis Ababa University, Addis Ababa, \\ Ethiopia; ${ }^{3}$ Department of Pathology, \\ College of Health Sciences, Addis Ababa \\ University, Addis Ababa, Ethiopia
}

Correspondence: Mohammed Mehdi Department of Biochemistry, College of Health Sciences, Addis Ababa University, P.o.box: 9086, Addis Ababa, Ethiopia Tel +251910066838

Email mohammed.mehdi@aau.edu.et
Background: Cellular proteases are thought to increase the likelihood of cancer cell infiltration and metastasis by degrading constituents of the extracellular matrix (ECM). Measuring activities of these proteases may be used as tumor markers for early diagnosis, prognosis, and as a possible target for treatment plan.

Objective: The aim of the current study is to evaluate cysteine cathepsins (CTSK and CTSL) and matrix metalloproteases-2 (MMP-2) and 9 (MMP-9) activities in human breast tumor tissue.

Methods: A comparative cross-sectional study plan was devised to study the enzymatic activities ofCTSK and CTSL andMMP-2 and MMP-9 via zymographic detection method. Sites of tissue sample collection were St Paul's Millennium Medical College, Menelik II Hospital and Zewditu Memorial Hospital, Addis Ababa, Ethiopia. A total of 36 breast cancer patients were recruited and tissue samples were collected for the study.

Results: Activities of CTSK and CTSL were significantly elevated in cancerous tissue than the adjacent normal non-cancerous breast tissue of the same patients $(n=36, p \leq 0.05)$. Also, activities ofMMP-2 and MMP-9 were increased significantly in tumor tissues than normal tissues $(\mathrm{n}=36, \mathrm{P} \leq 0.05)$.

Conclusion: It is found that there are different patterns of protease enzymatic activity expression between normal and tumor tissue using zymography. Compared with normal tissue samples, the protease enzymatic activity in cancerous tissue is higher. Thus, tissue proteases can be used in conjunction with histological techniques to identify patients in the same clinical group.

Keywords: breast cancer, cathepsin K; CTSK, cathepsin L; CTSL, matrix metalloprotinease-9; MMP-9, matrix metalloprotinease-2; MMP-2

\section{Introduction}

Breast cancer is a heterogeneous and multifactorial disease. It affects humans, especially women, with a high frequency of morbidity and mortality. ${ }^{1}$ Globally the prevalence and incidence of cancer are increasing at an alarming rate. Around 14.1 million are affected by cancer and 8.2 million people died as a result of cancer and cancer-related complications in $2012 .^{2}$

Women who are diagnosed with late-stage breast cancer in developing countries is usually due to the lack of early biomarkers for diagnosis and management. ${ }^{3}$ The only effective breast cancer screening method so far is mammography. Mammography screening is expensive for developing countries, but long lasting public screening programs are affordable and feasible in countries with a good health infrastructure. ${ }^{3,4}$ 
Proteolytic activities of enzymes are required in order to damage parts of the extracellular matrix (ECM) and the basement membrane and cause invasion and metastasis of tumor cells. The degradation of the ECM looks to smooth tumor cell exodus causative to the metastatic distribution of malignant abnormal cells to distant parts of the body from the primary location of the cancer. ${ }^{5}$

Cysteine cathepsins belong to the family of papain-like cysteine peptidases. Recently, it has been identified in the local microenvironment which is secreted extracellularly in to it and contributes to ECM remodeling. ${ }^{6}$ Platt's study showed that clear bands of proteolytic activities of cathepsins K (CTSK) and L (CTSL) in tissue preparations once SDS-PAGE zymography (gelatin substrate) in non-denaturing and non-reducing in settings of optimal cathepsin and matrix metalloprotinease (MMP) renaturing activity. The information of zymogram is provided quantitatively via densitometric analysis. ${ }^{7,8}$

The latest evidence recommends that MMP-2 and MMP9 may also be associated with breast cancer commencement and development through complex communications with the principle oncogenes and tumor-silencer genes engaged with the beginning phase of tumorigenesis. ${ }^{9}$ For instance, there is an increased expression of MMP-2 subsequently of transfection of MCF-10A breast tumor cell line with either c-erbB2 or c-ras, ${ }^{10}$ whereas, there is an increased production of MMP-9 as a result of transfection of MCF-7 cell line with the gene PEA- $3 .{ }^{10}$ Because of the vital role of MMPs in tumorigenesis, several researcher have proposed MMP-2 and or MMP-9 as helpful prognostic markers. ${ }^{10}$ Ongoing work with breast cancer patients has recommended that MMP-2 negativity might be connected with an ideal anticipation of node-negative breast carcinoma, ${ }^{11}$ and a high enzymatic activity of MMP-9 in a plasma sample of breast cancer patients are related with a poor general survival rate. ${ }^{5}$

The zymography method has a number of important features and is convenient when compared to alternative methods such as enzyme-linked immunosorbent assay, which requires costly equipment and reagents, such as antibodies and several proteases. This shows activity on the same substrate that can be detected and quantified on a single gel. The MMPs $(10 \mathrm{kDa})$ is cleaved from cells in a proteolytically inactive form called zymogen which is a higher molecular weight than the activated form. Since zymogen are activated at the time of the renaturation process after gel electrophoresis, both active and inactive forms can be measured and detected on zymograms. Moreover, MMPs in solution are usually associated with their inhibitors of metalloproteases (TIMPs) and disassociated during electrophoresis, as result they do not interfere with the detection of the enzymatic activity. ${ }^{12}$

As the incidences of breast cancer continues to rise in less developed countries, finding and using cheap and inexpensive quantitative assays to detect the disease early is urgently needed. Therefore, the current study aimed to measure the enzymatic activities CTSK, CSTL, MMP-2, and MMP-9 using zymography.

\section{Methodology}

A relative cross-sectional study design was used to study zymographic detection of cysteine cathepsins and MMP activities in human breast cancer tissue at the St Paul's Millennium Medical College, Menelik II Hospital, and Zewditu Memorial Hospital, in Addis Ababa, Ethiopia. These are major hospitalstreatingcancer patients and offering mastectomy servicesfor breast cance patients. All participants with histologically-confirmed breast cancer and who had breast excisional surgery were involved in the study.

Thirty-six breast cancer patients were recruited for the study. Both tumor (cancerous) and adjacent non-cancerous normal tissue samples were harvested from the same patient by surgeons. A total of 72 tissue samples were run for zymography and duplicated. The sociodemographic data were collected by using structured closed-ended, as well as open-ended, self-administered questionnaires. The TNM staging was done according to the American Joint Committee on Cancer TNM staging system (AJCC). ${ }^{13,14}$

\section{Tissue Sample Collection and Homogenization}

Tissue sample collection was performed by the attending surgeon and homogenization was carried out according to Smith and Xu, 2012. ${ }^{15}$ Immediately after surgery (within 10 minutes), fresh tissue samples were collected from eligible participants. All specimens were soaked in phosphate-buffered saline (PBS) pH-7 and preserved, then transported to Tikur Anbessa Specialized Hospital (TASH) with cold chain and frozen at $-80{ }^{\circ} \mathrm{C}$ refrigerator until analysis.

All samples were treated adequately to maintain the function of the enzymes and used immediately after collection or frozen at $-80^{\circ} \mathrm{C}$. During the time of laboratory analysis, the fat-free tissue was crushed $(\sim 50 \mathrm{mg})$ into small fragments with a scalpel in a sterile Petri dish. Cold lysis buffer of $250 \mu \mathrm{L}$ with protease inhibitor (leupeptin) freshly added on the top of minced tissue specimens. Then, the tissue was homogenized with the lysis buffer (5 mM EGTA, $20 \mathrm{nM}$ 
Tris- $\mathrm{HCl}$ at $\mathrm{pH} 7.5,150 \mathrm{mM} \mathrm{NaCl}, 10 \mathrm{mM} \mathrm{NaF}, 20 \mathrm{mM} \beta$ glycerol-phosphate, $1 \%$ Triton X-100, $1 \mathrm{mM}$ sodium orthovanadate, $0.1 \%$ Tween-20, $0.1 \mathrm{mM}$ leupeptin newly pipetted) with a stainless steel spatula, then the supernatant aliquot was collected and incubated on ice for at least 5 minutes. The aliquot was mixed with Vortex and centrifuged at 10,000 rpm for 10 minutes at $4^{\circ} \mathrm{C}$ in a microcentrifuge. The collected supernatant was used to measure protein concentration so, normalization of protein concentration was done to $1 \mu \mathrm{g}$ per $\mu \mathrm{L}$, with bicinchoninic acid (BCA) total protein assay, and vortexed with $5 \mathrm{X}$ sample buffer. Finally, the sample loaded, which equal amount of protein per lane on freshly prepared gel electrophoresis.

\section{Multiplex Zymography Principle}

Cathepsin zymography was done based on the technique of Chen and Platt. ${ }^{16}$ Mini Gel was made from short glass plates and is used to produce small amounts of Protein 3 multi-casting (Bio-Rad) standard size gels approximately $92 * 68 \mathrm{~mm}$ (width * height). The standard zimmogram were washed $3 \mathrm{X}$ for $10 \mathrm{~min}$ each in a renaturing buffer (20\% glycerol, 65mM Tris buffer, $\mathrm{pH} 7.4$ ), balanced in assay buffer (phosphate buffer, 1mM EDTA, pH 6, and $2 \mathrm{mM}$ DTT) for 30 minutes before $24 \mathrm{hr}$ incubation period at $37^{\circ} \mathrm{C}$ in the freshly made assay buffer. To stimulate the mini-zimo gel enzymes, they were rinsed 3 times each in a vacuum chamber for 5 minutes and balanced by assay buffer which isbefore the assay buffer incubation.

MMP zymography was carried out similarly, except renaturation, it was performed by $2.5 \%$ Triton-X100 and incubated in $50 \mathrm{mM}$ Tris $\mathrm{HCl}$, pH 7.4; $10 \mathrm{mM}$ calcium chloride; $50 \mathrm{mM} \mathrm{NaCl} ; 0.05 \%$ Triton - X100 test buffer overnight.

\section{Quantitation of Gel}

Gels were quantitated using ImageJ-NIH software. The gel image was rotated to show white bands on a black background. The optical dimensions, molecular weight, and area of each band were measured.

\section{Data Analysis and Interpretation}

Information obtained from zymographic quantification of the enzymatic activity of CTSK, CTSL, and latent and matured MMP-2 and MMP-9 were plotted via MS Excel program for total protein test. The MS Excel were exported to IBM-SPSS version 21 program for statistical analyses of unpaired Student's T-test and one-way ANOVA with Post Hoc, and Pearson association analysis. The appropriateness of data to normal distribution was evaluated by the technique of
Kolmogorov and Smirnov. This normality test measures the inconsistency among the examined distributions of data and an ideal Gaussian distribution; the test gives a P-value $>0.05$ that is considered as acceptable for further analysis. However, all parameter's distributions were examined and log transformed for their fitness with a normal distribution. So as to assess the significance of differences amongst cancer patients and control subjects, an unpaired Student's T-test and one-way ANOVA was applied. If $P$. value is less than or equal to 0.05 , it is considered as statistically significant.

\section{Ethical Concern}

The study was conducted in accordance with the Declaration of Helsinki. First, Ethical clearance was issued from the research and an ethical review committee of the biochemistry department of AAU with a protocol no. SOM/BCHM/129/ 2006 and IRB protocol no.068/18/biochem. Then, all study participants were well informed and signed the informed consent form. The participants were also told about the privacy and confidentiality of their personal information and will not be disclosed to a third party by any means.

\section{Result}

\section{Socio-Demographic Features of the Breast Cancer Patients}

A total of 36 (36 tumor tissues and 36 adjacent normal tissues) participants were recruited and studied. The age range of patients was from 24 to 83 with a median age of 39 years. The peak age distribution of study participants was between 30 to 39 years $(n=10,27.8 \%)$.

Out of the 36 study participants, 17 were (47.22\%) fromAddis Ababa city; 30.56\% were from the Oromia region; $11.11 \%$ were from Amhara; and 11\% were from the southern nation, nationality, and people region (SNNPR). Of the total study participants, $61.1 \%(\mathrm{n}=22)$ came from urban regions of Ethiopia. The rest 38.9\% $(\mathrm{n}=$ 14) came from the rural part of Ethiopia.

The educational status of the study participants showed that $36.1 \%(\mathrm{n}=13)$ of them did not get a formal education, $55.6 \%(\mathrm{n}=20)$ were at the high school level and the remaining $8.3 \%(n=3)$ were attending college at the time of data collection. The marital status of participants was also assessed and $72.2 \%(n=26)$ were married, $16.7 \%(n=6)$ were widowed and $11.1 \%(n=4)$ were single during data collection.

The time duration of onset of symptom to the day of surgery was between 2 and 48 months (median 5.5 months). None of the study participants have a history of alcohol 
consumption and cigarette smoking in their lifetime. However, among the study participants $(30.6 \%(\mathrm{n}=11)$ took oral contraceptive pills (OCP) and the remaining participants $(n=25$, $69.4 \%$ ) had no experience of OCPs in their lifetime.

\section{Clinical and Pathological Results}

Of the total study participants, 27 (75\%) of them were nodepositive. The histopathological result showed that 34 (94.4\%) had infiltrative ductal carcinoma. The number of women who had had a child and had all breastfed was $n=26$. From the clinical and mammography result, we found an increasing number of patients with upper outer quadrant (UOQ) breast tumors. That is, $20(55.6 \%)$ patients presented with a UOQ breast lump. Among the 36 breast cancer patients, 19 patients had histologically confirmed right breast cancer (Table 1).

\section{Cathepsin K and Cathepsin L Expression}

Cathepsin zymography detection method detects a mature CTSK and CTSL activity after loading an equal amount of sample $20 \mu \mathrm{L}(20 \mu \mathrm{g} / \mu \mathrm{L})$ of sample aliquot to each gel wells. CTSK and CTSL seemed as zymographically active

Table I Clinical and Physical Characteristics of Breast Cancer Patients $(n=36)$

\begin{tabular}{|l|l|l|}
\hline Characteristics & Number $\mathbf{( N}=\mathbf{3 6})$ & Proportion \% \\
\hline $\begin{array}{l}\text { Nodal status } \\
\text { Positive }\end{array}$ & 27 & 75.0 \\
Negative & 9 & 25.0 \\
\hline Histological type & & \\
Infiltrative ductal & 34 & 94.4 \\
Lobular & 2 & 5.6 \\
\hline Breast feeding & & \\
Breast fed & 26 & 72.2 \\
Non breast fed & 10 & 27.8 \\
\hline Location of breast cancer & & \\
UOQ & 20 & 55.6 \\
UIQ & 5 & 13.9 \\
LOQ & 6 & 16.7 \\
LIQ & 2 & 5.6 \\
Central & 3 & 8.3 \\
\hline Breast cancer site & & \\
Right & 19 & 52.8 \\
Left & 16 & 2.8 \\
Both & 1 & \\
\hline Tumor size (mean \pm SD) & & \\
Length & $6.49 \mathrm{~cm} \pm 3.90 \mathrm{~cm}$ & \\
Width & $6.30 \mathrm{~cm} \pm 3.57 \mathrm{~cm}$ & \\
\hline
\end{tabular}

bands at particular molecular weights from breast cancer samples (Figure 1); mature CTSL band looked adjacent to the $21 \mathrm{kDa}$ size and CTSK by the side of $37 \mathrm{kDa}$. Multiplex zymography does not demonstrate a clearly visible white band for normal tissue specimen even after 40 $\mu \mathrm{g} / \mu \mathrm{L}$ sample loaded to each gel wells.

\section{MMP-2 and MMP-9 Expression}

Due to differences in the molecular mass of gelatinases MMP-2 and MMP-9 gelatin, gelatin zymography distinguish and separate MMP-2 and MMP-9 in a latent and active forms. In cancerous and non-cancerous tissues of breast samples, the following four lysis bands were observed in the samples: $92 \mathrm{kDa}$, which is parallel to hidden MMP-9; $84 \mathrm{kDa}$, active MMP-9; $72 \mathrm{kDa}$, hidden MPP-2, and finally 68 kDa, active MMP-2 (Figure 2).

\section{Cathepsin and MMPs' Activities Between Normal and Tumor Breast Tissue Specimens}

Statistically significant proteases enzymatic activities were detected in breast tumor tissue specimens as compared to adjacent normal breast tissue specimens (Table 2).

The gelatinolytic activity of cathepsins and MMPs were analyzed within two age groups. The age was categorized into $\geq 50$ and $<50$ years. The result was compared with the same age group between normal and tumor tissues. In women aged $<50$ years $(n=22)$. The mean $(X)$ and standard error (SE) of the mean in tumor and normal tissue, respectively, were: CTSK $3766 \pm 1262$ and $747 \pm 201$, CTSL $2895 \pm 590$ and 581 \pm 119 , pro-MMP-9 2998 \pm 379 and $1676 \pm 457$, MMP-9 $6407 \pm 771$ and $2741 \pm 693$, pro-MMP-2 $1444 \pm 296$ and $1415 \pm 422$ finally MMP-2 $3956 \pm 544$ and $1857 \pm 431$.

In women aged above and $\geq 50$ years, the mean $(X)$ and $\mathrm{SE}$ of the mean in tumor and normal tissue specimens respectively, were: CTSK $2622 \pm 586$ and $738 \pm 232$, CTSL $3126 \pm 671$ and 414 \pm 84 , pro-MMP-9 $2632 \pm 530$ and $1091 \pm 384$, MMP-9 8519 \pm 1147 and $3283 \pm 887$, pro-MMP$21170 \pm 268$ and $2133 \pm 583$ finally MMP-2 $2208 \pm 627$.

In the age group above 50 years activities of CTSL and CTSK on tumor tissue were elevated significantly when compared to adjacent non-cancerous normal tissue $(\mathrm{P} \leq 0.05)$.

The comparison result did not show significant activity of cathepsins and MMPs between tumor tissues of women aged $<50$ and $\geq 50$ years of age. 


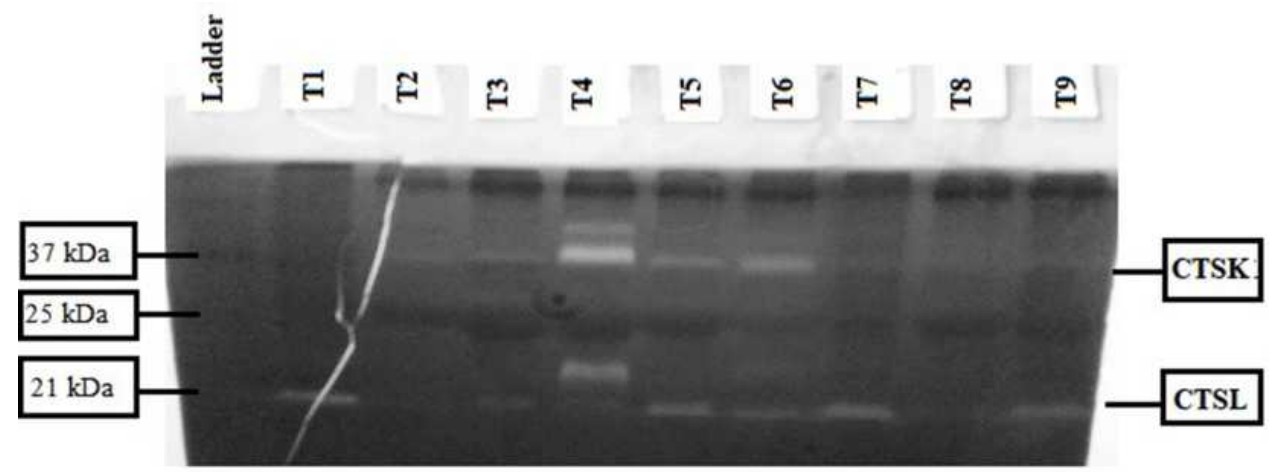

Figure I Cathepsin zymogram of tissue specimens in breast cancer. It shows $20 \mu \mathrm{g}$ tumor tissue specimens from patients with breast cancer biopsies were loaded for zymography. Cathepsin K band were visible at $37 \mathrm{kDa}$ and cathepsin $\mathrm{L}$ at $21 \mathrm{kDa}$ based on the standards molecular weight of CTSK and CTSL shown on the left panel (ladder).
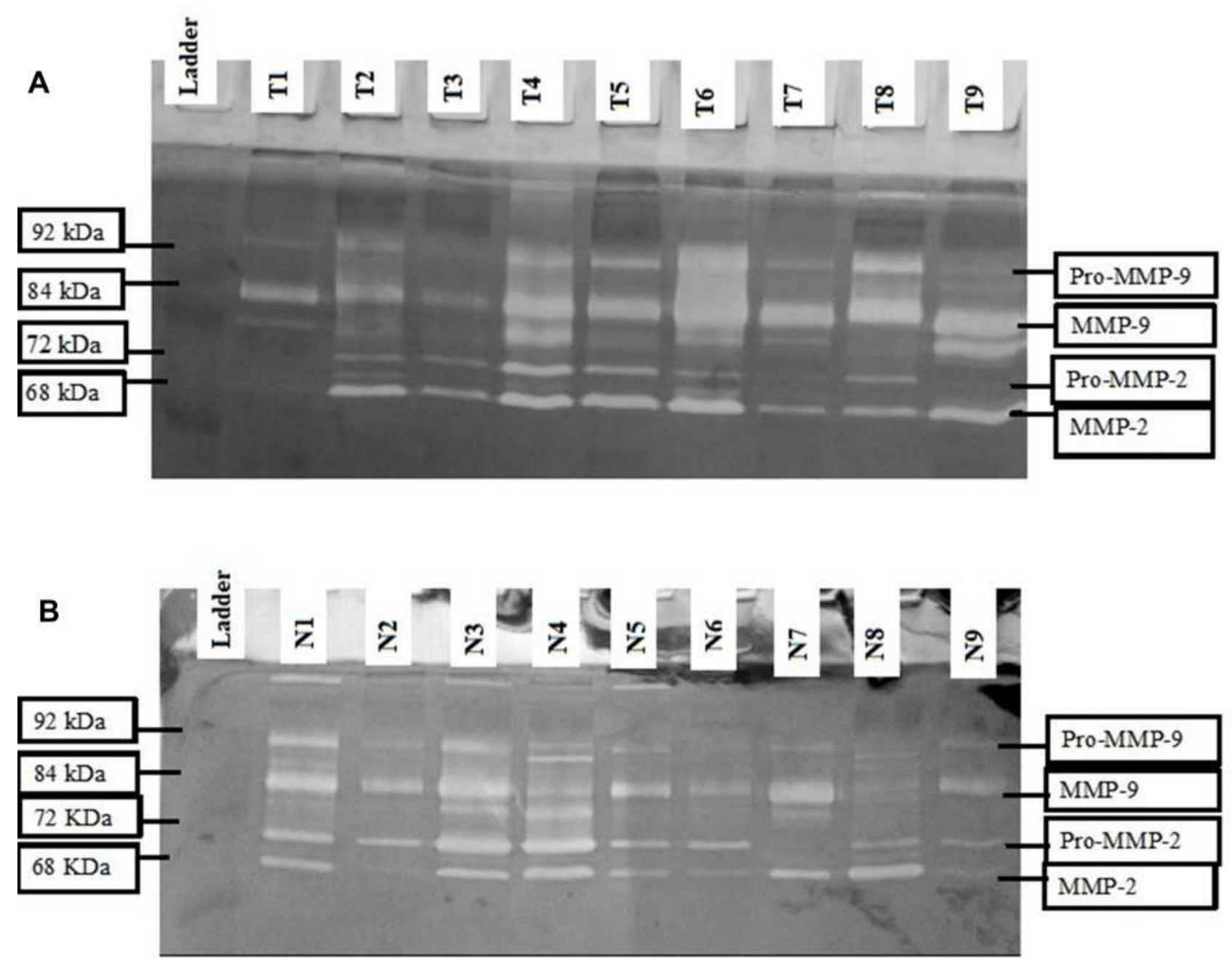

Figure 2 Matrix metalloproteinases zymogram in breast cancer tissue specimens. A $20 \mu \mathrm{g}$ of tumor (A) and adjacent normal (B) breast tissue from breast cancer patient biopsies were loaded for zymography. Pro-MMP-9 band is visible at 92 kDa, MMP-9 at 84 kDa, pro-MMP-2 at 72 kDa and MMP-2 at 68 kDa. 
Table 2 Mean, Standard Error of Mean of MMPs and Cathepsins (Pixel) Between Normal and Tumor Breast Tissue Specimens $(n=36)$

\begin{tabular}{|l|c|c|c|c|l|}
\hline \multirow{2}{*}{ Variables } & \multicolumn{2}{|c|}{ Tumor } & \multicolumn{2}{c|}{ Normal } & \multirow{2}{*}{ P-value } \\
\cline { 2 - 5 } & Mean & SEM & Mean & SEM & \\
\hline Pro-MMP-9 & 2856 & 307 & 1225 & 217 & 0.001 \\
MMP-9 & 7229 & 662 & 3911 & 534 & 0.001 \\
Pro-MMP-2 & 1338 & 207 & 1558 & 328 & 0.247 \\
MMP-2 & 3504 & 392 & 1393 & 308 & 0.001 \\
CTSK & 3321 & 801 & 744 & 150 & 0.001 \\
CTSL & 2985 & 439 & 516 & 80 & 0.001 \\
\hline
\end{tabular}

The comparison of cathepsins and MMPs' gelatinolytic activity in mothers with breast cancer aged $<25$ years at first live birth between tumor and normal breast tissue specimen showed significantly increased activity in tumor tissue (Table 2).

The comparison of cathepsins and MMPs' gelatinolytic activity in mothers with breast cancer $\geq 25$ years at first live birth between tumor and normal breast tissue specimen showed non-significant activity in tumor tissue (Table 3). However, MMP-2 showed significant gelatinase activity (Table 4). Also, in women who deliver their first live birth aged less than 20,cathepsin activity was significantly higher when compared to the same women in the age range of normal tissue specimens $(\mathrm{P}<0.001)$. There was no significant activity of these enzymes between the intergroup.

In Table 5, latent MMP-9 showed highly significant activities among women who experienced their first menarche $\geq 14$ when compared between tumor and normal tissue specimens $(\mathrm{P}<0.001)$. Also, in premenopausal women there was highly significant activity of pro-MMP-9 between tumor and normal breast tissue specimens (Table 5).

In Table 6 CTSL, pro-MMP-9, and MMP-2 showed highly significant activity among women who experienced their first menarche $\leq 14$ years when compared between tumor and normal tissue specimens $(\mathrm{P}<0.001)$. Similarly, in premenopausal women there was highly significant activity of pro-MMP-9 between tumor and normal breast tissue specimens (Table 6).

Accordingly, pro-MMP-2, and other enzymes like CTSK, pro-MMP-9, CTSL, MMP-2 and MMP-9, were significantly expressed in a gelatin zymography-band.

The gelatinolytic activity of these enzymes in tumor and normal tissue specimen respectively was expressed as follows in pixels $(n=24)$ : enzyme activity between tumor and normal tissues in women age $<50$ years; in premenopausal breast
Table 3 Activities of Cathepsin and MMPs (Pixel) in Mothers Age $<25$ During First Live Birth $(n=20)$

\begin{tabular}{|c|c|c|c|c|}
\hline \multirow[t]{2}{*}{ Variables } & \multirow[t]{2}{*}{$\mathbf{n}$} & Tumor & Normal & \multirow[t]{2}{*}{ p-value } \\
\hline & & Mean \pm SEM & Mean \pm SEM & \\
\hline CTSK & 20 & $3272 \pm 928$ & $834 \pm 217$ & 0.015 \\
\hline CTSL & 20 & $3728 \pm 649$ & $550 \pm 111$ & 0.001 \\
\hline Pro-MMP-9 & 20 & $3387 \pm 487$ & $1106 \pm 272$ & 0.001 \\
\hline MMP-9 & 20 & $7976 \pm 844$ & $3485 \pm 573$ & 0.001 \\
\hline Pro-MMP-2 & 20 & $1315 \pm 300$ & $1912 \pm 463$ & 0.286 \\
\hline MMP-2 & 20 & $3591 \pm 610$ & $1839 \pm 468$ & 0.028 \\
\hline
\end{tabular}

Table 4 Non-Significant Activity Change of Cathepsin and MMPs (Pixel) in Mothers Age $\geq 25$ During First Live Birth $(n=6)$

\begin{tabular}{|c|c|c|c|c|}
\hline \multirow{2}{*}{ Variables } & \multirow[t]{2}{*}{$n$} & \multirow{2}{*}{$\begin{array}{l}\text { Tumor } \\
\text { Mean } \pm \text { SEM }\end{array}$} & \multirow{2}{*}{$\begin{array}{l}\text { Normal } \\
\text { Mean 士SEM }\end{array}$} & \multirow[t]{2}{*}{ p-value } \\
\hline & & & & \\
\hline CTSK & 6 & $1870 \pm 485$ & $949 \pm 500$ & 0.216 \\
\hline CTSL & 6 & $2793 \pm 1011$ & $361 \pm 106$ & 0.038 \\
\hline Pro-MMP-9 & 6 & $2914 \pm 290$ & $|629 \pm 64|$ & 0.098 \\
\hline MMP-9 & 6 & $745 I \pm 2354$ & $5215 \pm 1588$ & 0.449 \\
\hline Pro-MMP-2 & 6 & $1990 \pm 445$ & $1041 \pm 999$ & 0.406 \\
\hline MMP-2 & 6 & $3953 \pm 1030$ & $490 \pm 432$ & 0.011 \\
\hline
\end{tabular}

Table 5 Activities of Cathepsin and MMP-9 (Pixel) in Late Menarche Women with Breast Cancer. Menarche $\geq 14$ Years (n $=14)$

\begin{tabular}{|c|c|c|c|c|}
\hline \multirow[t]{2}{*}{ Variables } & \multirow[t]{2}{*}{$\mathbf{n}$} & Tumor & Normal & \multirow[t]{2}{*}{ p-value } \\
\hline & & Mean \pm SEM & Mean \pm SEM & \\
\hline CTSK & 14 & $3248 \pm 1230$ & $629 \pm 152$ & 0.044 \\
\hline CTSL & 14 & $3200 \pm 891$ & $465 \pm 95$ & 0.005 \\
\hline Pro-MMP-9 & 14 & $2707 \pm 498$ & $1496 \pm 327$ & 0.052 \\
\hline MMP-9 & 14 & $7485 \pm|24|$ & $3631 \pm 679$ & 0.011 \\
\hline Pro-MMP-2 & 14 & $1346 \pm 333$ & $1926 \pm 556$ & 0.379 \\
\hline MMP-2 & 14 & $2615 \pm 492$ & $1866 \pm 562$ & 0.325 \\
\hline
\end{tabular}

cancer patients showed CTSK $3786 \pm 1155$ and $775 \pm 193$, CTSL $2859 \pm 556$ and $599 \pm 113$, pro-MMP-9 $3024 \pm 377$ and $1231 \pm 248$, MMP-9 $6413 \pm 742$ and $4114 \pm 639$, pro-MMP-2 $1547 \pm 280$ and $1228 \pm 347$, MMP-2 $4032 \pm 503$ and $895 \pm 245$. The activity is increased, but not significant $(\mathrm{P}>0.05)$.

The gelatinolytic activity of these enzymes in tumor and normal tissue specimen respectively was expressed as follows in pixels $(n=12)$ : enzyme activity between tumor and normal tissues in postmenopausal breast cancer patients showed CTSK $2391 \pm 661$ and $681 \pm 243$, CTSL $3237 \pm 735$ and $350 \pm 66$, pro-MMP-9 $2519 \pm 537$ and $1213 \pm 438$, MMP-9 $8660 \pm 1234$ and $3506 \pm 998$, proMMP-2918 \pm 238 and $2220 \pm 681$ and finally MMP-2 2450 
Table 6 Activity Cathepsins and MMPs (Pixel) in Early Menarche Women with Breast Cancer. Menarche $<14$ Years $(n=21)$

\begin{tabular}{|c|c|c|c|c|}
\hline \multirow{2}{*}{ Variables } & \multirow[t]{2}{*}{$n$} & \multirow{2}{*}{$\begin{array}{l}\text { Tumor } \\
\text { Mean } \pm \text { SEM }\end{array}$} & \multirow{2}{*}{$\begin{array}{l}\text { Normal } \\
\text { Mean ISEM }\end{array}$} & \multirow[t]{2}{*}{ p-value } \\
\hline & & & & \\
\hline CTSK & 21 & $3479 \pm \mid 121$ & $846 \pm 236$ & 0.027 \\
\hline CTSL & 21 & $2859 \pm 482$ & $569 \pm 121$ & 0.001 \\
\hline Pro-MMP-9 & 21 & $3002 \pm 4 \mid 4$ & $918 \pm 266$ & 0.001 \\
\hline MMP-9 & 21 & $6977 \pm 798$ & $3783 \pm 74 \mid$ & 0.006 \\
\hline Pro-MMP-2 & 21 & $|376 \pm 28|$ & $1180 \pm 397$ & 0.689 \\
\hline MMP-2 & 21 & $4199 \pm 542$ & $1082 \pm 369$ & 0.001 \\
\hline
\end{tabular}

\pm 505 and $2389 \pm 722$. It showed that it is significantly amplified $(\mathrm{P}<0.05)$.

The activity of cathepsins and MMPs were not significantly increased between multigravida and nulliparous women. Parity does not change cathepsin and the MMPs' level of activity. The mean \pm SEM of enzymatic activity between multigravida and nulliparous women in tumor tissue ( $\mathrm{n}=26$ multigravida, $\mathrm{n}=10$ nulliparous) respectively were: CTSK 2949 \pm 727 and 4290 \pm 2243 , CTSL 3512 \pm 547 and $1613 \pm 499$, pro-MMP-9 $3278 \pm 380$ and 1758 \pm 930 , MMP-9 $7855 \pm 821$ and $5600 \pm 930$, pro-MMP-2 $1470 \pm 255$ and $992 \pm 336$ and finally MMP-2 $3674 \pm 517$ and $3063 \pm 428(\mathrm{P}>0.05)$.

The comparison of CTSK and CTSL between tumor and normal tissue showed that both were significantly higher in tumor tissue specimens. Tumor MMP-2 was 4 times increased and MMP-9 activity were 7 times higher than normal tissue. The activity of all latent and mature MMPs were higher in tumor tissue as compared to nontumor tissue (Figure 3).

\section{Tumor Size-Specific Difference of Cathepsins and MMPs in Breast Cancer}

The present study showed that there was a close association between human breast cancer and functional activity of CTSK and CTSL. Both were significantly higher in cancerous tissue than normal non-cancerous tissue specimens (Figure 4).

The current study compared MMPs' enzymatic activity with different tumor sizes of breast cancer. Both latent and mature MMPs' enzymatic activity were analyzed and compared through stages II and III. The result of zymography band densitometry result was analyzed and the enzymatic activity of pro-MMP-9 were significantly increased between $\mathrm{T} 2$ tumor size and normal breast tissue specimens $(\mathrm{n}=17)$. As well as between T3 and normal breast tissue specimens $(\mathrm{n}=18)$ (Figure 5).

\section{Breast Cancer's Stage-Specific Difference in Cathepsins $\mathrm{K}$ and $\mathrm{L}$}

Using cathepsin zymography, cathepsin activity was also determined for any stage-specific alterations in breast cancer. Thirty-six different specimens were analyzed in different stage categories. Samples of stages II and III breast tumor tissue (TNM stage determined based on AJCC Staging Guide) and non-tumor tissues were collected and loaded and run for cathepsin zymography (Figure 6).

The pathology report from TASH showed that stage II and III breast cancer patients are dominant. The CTSK activity on stages II and III of the patients' illness were significantly higher (Figure 6A, $n=36$ ). CTSL enzymatic activity in stages II and III was statistically significant and higher than in non-tumor tissue (Figure 6B, $\mathrm{n}=36$ ).

\section{Breast Cancer's Stage-Specific Difference in MMPs' Enzymatic Activity}

In this study we were required to assess any stage-specific alterations in breast cancer MMPs' enzymatic activity utilizing MMPs' zymography measurement. Thirty-six different samples were analyzed in different stage category. Samples of each stage II and III breast cancer tissue (TNM staging based on AJCC staging guide) and nontumor tissues were collected from the same patient and loaded and run for MMPs' zymography (Figure 7).

Pro-MMP-9 activity significantly increased at stage III and stage II compared to normal tissue (Figure 7A, $\mathrm{P}<0.05$ ). It is essential to remind that for pro-MMP-2 enzymatic activity in all stages of tumor tissue were examined and was not significantly increased compared to the non-tumor breast tissue. The stage-specific difference of MMP-2 and MMP-9 tested for these enzymes indicates that there is noticeable increment in the enzymatic activity of MMP-9 between stage III and normal $(\mathrm{n}=36, \mathrm{P}<0.01)$ as well as between stage II and non-tumor breast tissues. $(\mathrm{n}=36, \mathrm{P}<0.05)$. However there is no significant increment detected between stages II and III of the activity of MMP-9. On the other hand, MMP-2 were significantly higher between stage III and normal breast tissue specimens $(\mathrm{n}=36, \mathrm{P}<0.001)$.

The current study observed that there was no positive or negative correlation between CTSK and CTSL as well as between MMP-9 and MMP-2, also between cathepsin and MMPs. Similarly, CTS and MMPs does not show significant difference through histological grade between normal and tumor tissue. 
(A)
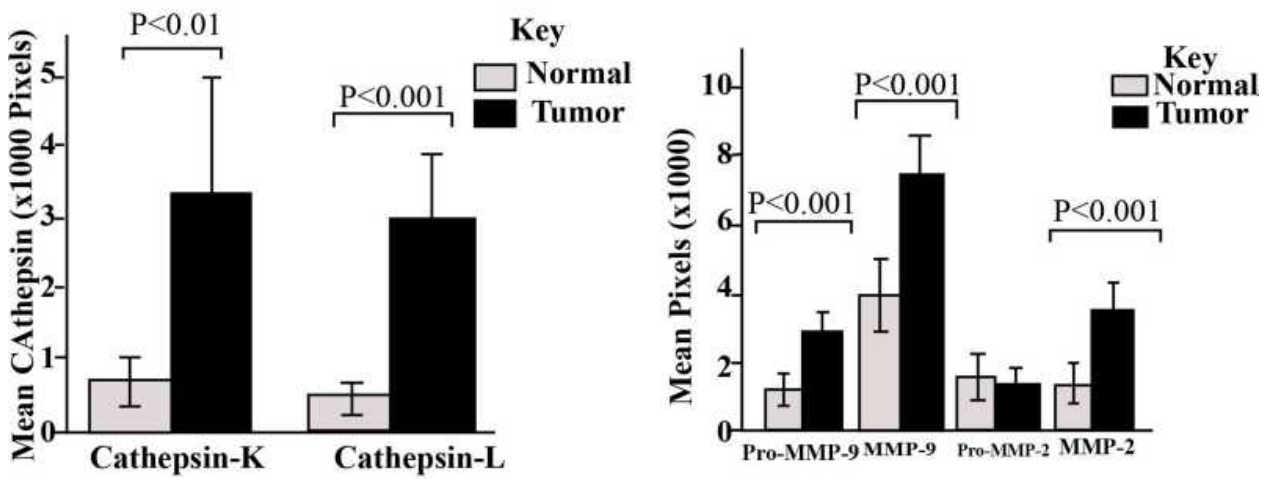

Figure 3 CTSK, CTSL, pro- and mature MMP-9 and MMP-2 enzymatic activities detected in the normal and tumor tissue of breast. (A) Each band densitometry of cathepsin (CTSK and CTSL) activities which were quantified on the gel. (B) Pro- and mature MMP-9 and MMP-2 activities which were quantified by band densitometry. All values are presented in pixel mean \pm SEM, times 1000 , of tumor compared to normal $(n=36)$.
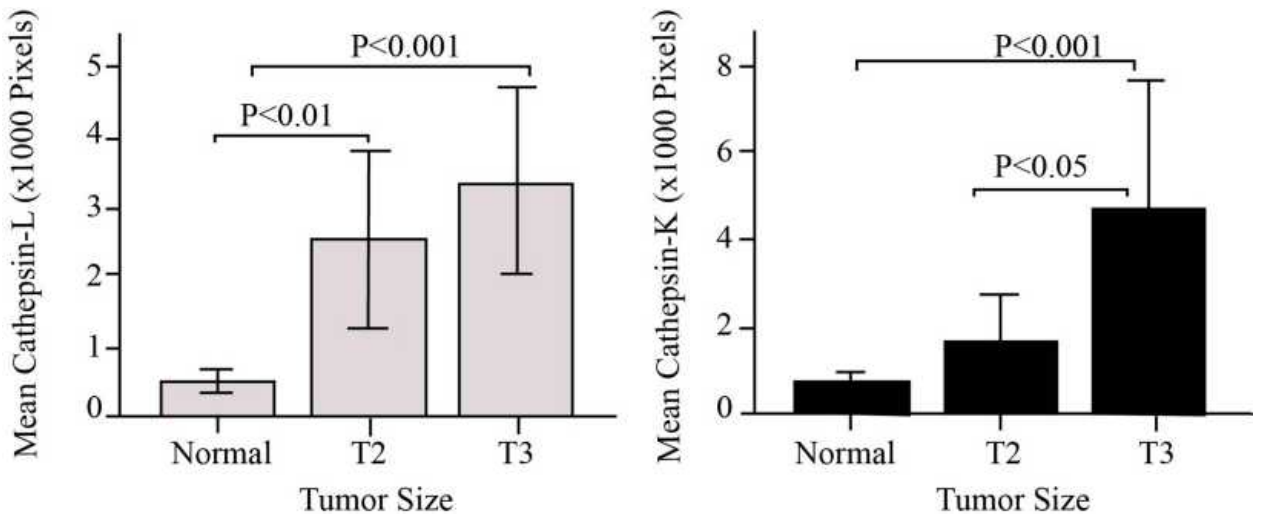

Figure 4 Tumor size-specific differences on cathepsin $\mathrm{K}$ and cathepsin $\mathrm{L}$ enzymatic activities in the breast cancer patients. Band densitometry were used to quantify the cathepsin $K$ and cathepsin $L$ enzymatic activities. All values are presented in pixel mean \pm SEM, times $1000(n=36)$.

\section{Discussion}

The incidence rate of breast cancer in women is strongly correlated with age, in Western countries being older in age is a risk for breast cancer which is linked with hormonal conditions. The present study's investigation showed that the incidences were higher in the age group of 30 to 39 years of age. In the UK, between 2010 and 2012, an average of $80 \%$ of breast cancers were detected and diagnosed in the over 50s, and $24 \%$ was detected and diagnosed in women aged 75 and over. ${ }^{17}$

The age-related prevalence of breast cancer cases in the UK showed that older age groups were most screened and diagnosed for breast cancer. However, breast cancer is becoming more common in women under the age of 40 . In the UK, among women aged 35-34, around 1300 breast cancer cases are diagnosed per year. Similarly the current study showed that breast cancer was also diagnosed in early premenopausal women. ${ }^{17}$

The clinical diagnoses of patients are done in different ways and one of the possible method is zymography; it has pronounced importance to clinical diagnoses along with histopathology owing to its numerous benefits: 1) antibody is not required and making it relatively cheap and speciesindependent; 2) after staining of the polyacrylamide gel, it is possible to confirm visually the separation protein's cathepsin and MMPs' identity through non-reducing electrophoretic migration; 3) quantitative analysis is possible via densitometry; and 4) multiplexed examination allows the peculiarity of latent and active CTSK and CTSL, as well as MMPs in tissue extraction or single cells. ${ }^{18}$

An additional screening parameter of pathological specimens was effectively demonstrated and studied by Platt; ${ }^{16}$ a multiplex zymography study to explore CTSK, CTSS, and 
1
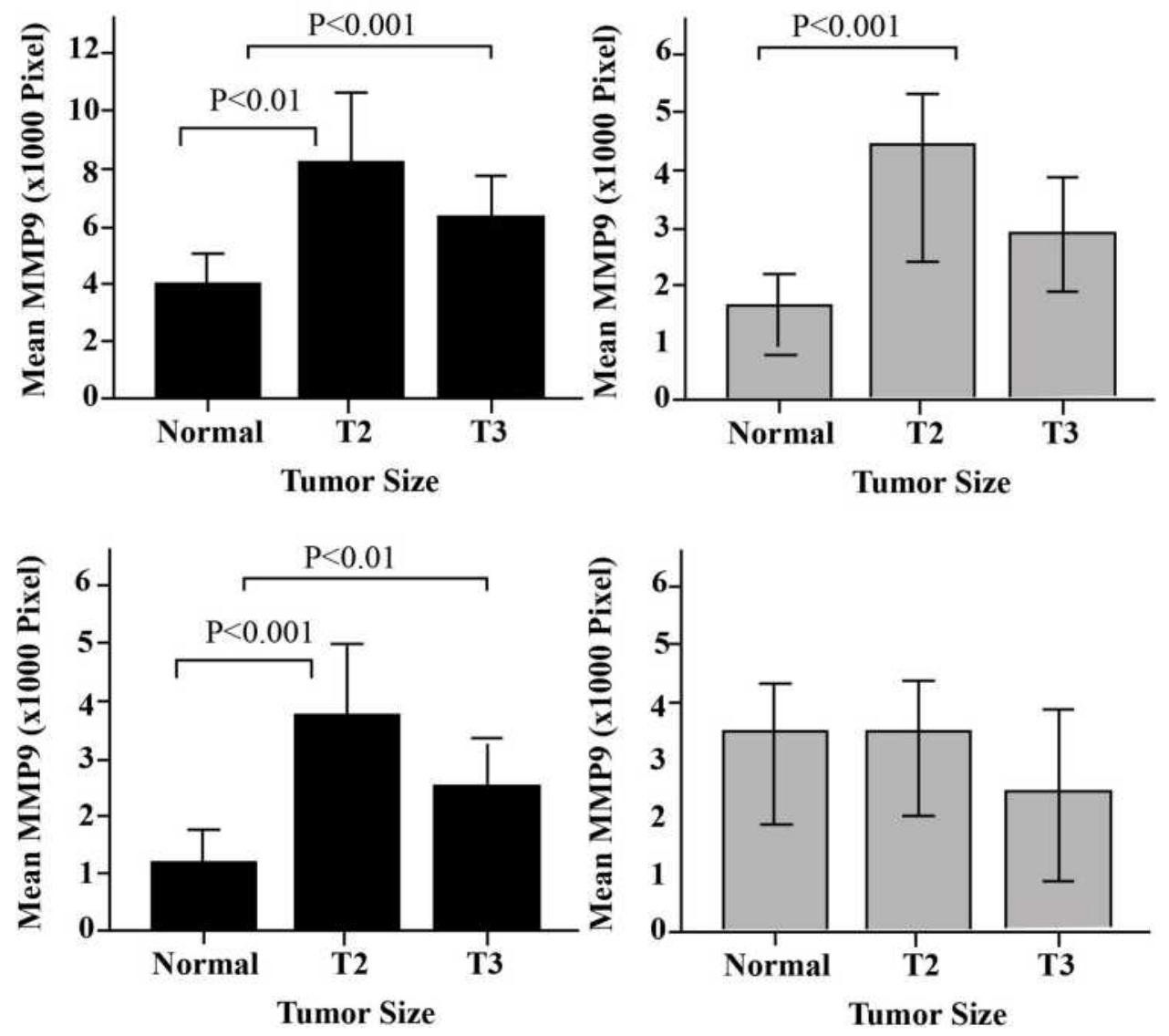

Figure 5 Tumor size-specific difference on pro- and mature MMP-9 and MMP-2 in the breast cancer patients. Pro- and mature MMP- 9 and MMP-2 band were quantified by semi-quantitative band densitometry. All values are presented in pixel mean \pm SEM, times $1000(\mathrm{~T} 2[\mathrm{n}]=17$ and T3 [n] = I8).
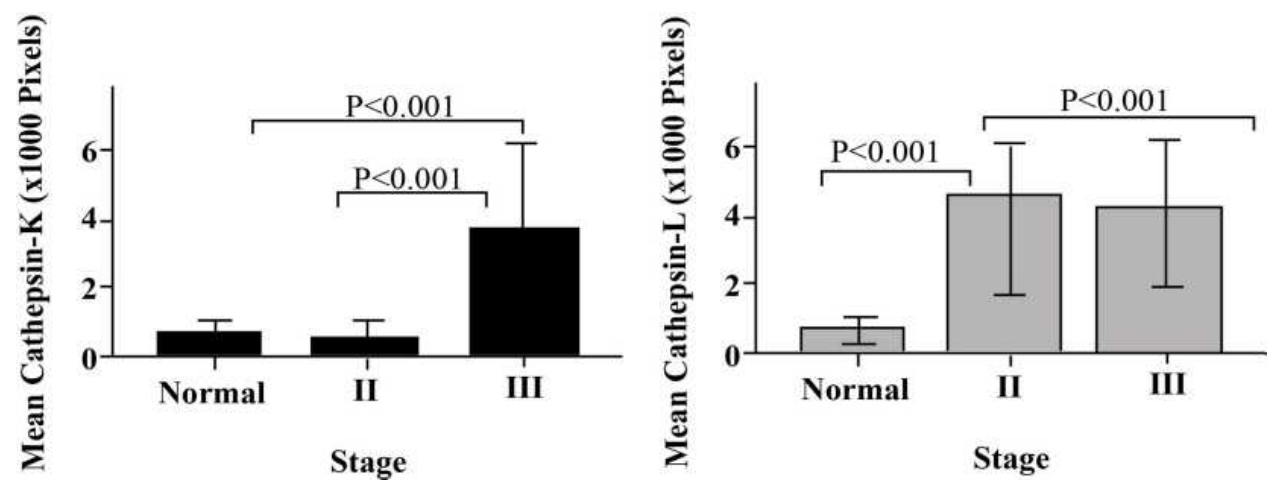

Figure 6 Stage-specific differences on CTSK and CTSL in the breast cancer patients. CTSK and CTSL activities were quantified by band densitometry. All values are presented in pixel mean \pm SEM (stage II $[\mathrm{n}]=8$ stage III $[\mathrm{n}]=28)$.

CTSL enzymatic activities in breast, cervical, and lung tissue at 3 different stages of cancer development. According to their study, zymography data were obtained after the clinical grading of biopsy tissue, which may supplement and meet gold standard histological methods to determine whether or not biopsy tissue is cancerous. ${ }^{16}$ 
(A)

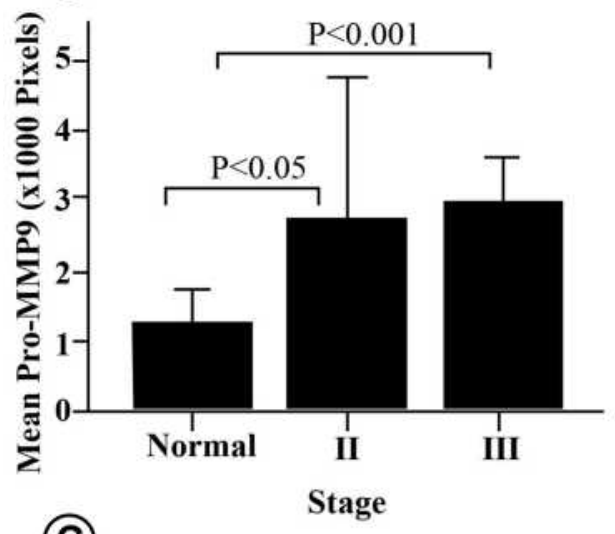

(C)

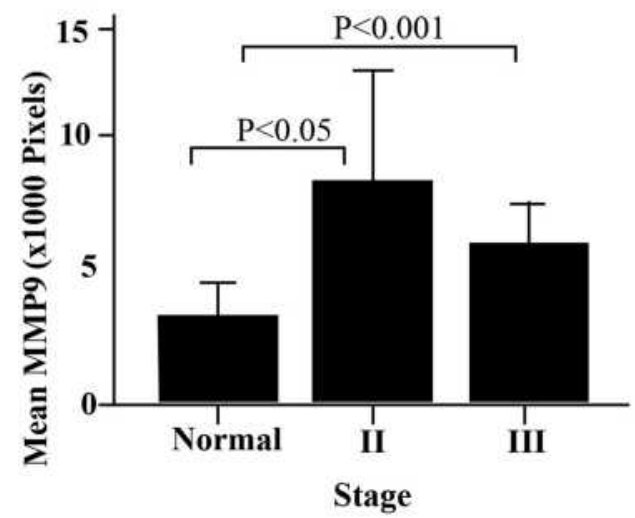

(B)

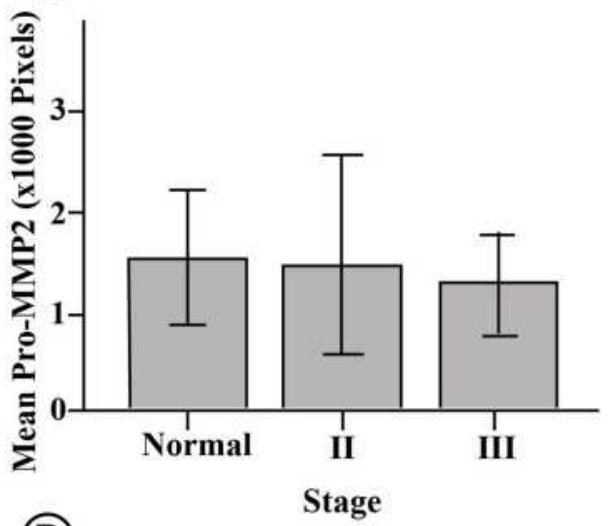

(D)

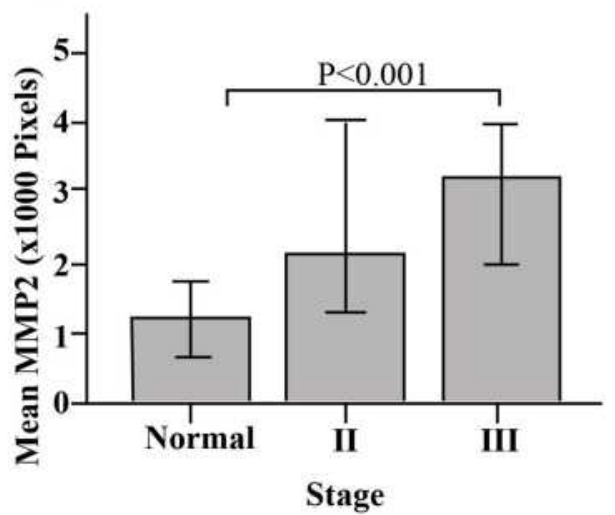

Figure 7 Stage-specific differences of MMPs in human breast cancer patients. (A) Pro-MMP-9 significantly higher in stage II and III compared to normal tissue. (B) Pro-MMP2 does not show any significant change across the stages. (C) MMP-9 significantly increased at stage III and II compared to normal tissue. (D) MMP-2 significantly increased between stage II and normal tissue. All values are presented in pixel mean \pm SEM, times $1000(n=36)$.

Distribution of cysteine cathepsin and MMPs in tumor and adjacent non tumor breast tissue specimens were investigated. The study also has studied lysis of tissue protein by proteolysis, one of the key processes occurred in cancer cell penetration, invasion and spread to other distant part of the body by metastasis. Under normal circumstances, proteolytic enzymes are highly controlled by means of specific proteinase inhibitors; the MMPs are controlled through TIMPs. After zymography, cathepsin and MMP lysis band of each samples (tumor and normal tissue) were determined by running in separate gel in zymogram. The major difference in proteinase enzyme expression was determined after gelatin impregnated SDS-PAGE gel electrophoresis.

It revealed that most of the 36 tissue samples from breast cancer patients showed sharp lysis bands of cathepsin and mature MMP-2, MMP-9 parallel to the proenzyme forms of MMP-2 and MMP-9, respectively, contrary to the 36 control samples which showed less distinct lytic bands for cathepsin and MMPs.
Cathepsin and MMPs' zymography revealed statistically non-significant, but shows negative correlation with the patient's age, however, CTSL has non-significant, but positive correlation. This phenomena may be because breast cancer at a younger age is more aggressive (triple negative) than at an older age. ${ }^{19}$ Aggressiveness of breast cancer is calculated by its tissue invasiveness and metastases to distant secondary organs; to do that, tumor cells and the surrounding stromal cells express increased levels of cathepsin and MMPs to degrade the collagen, which is a component of basement membrane for intravasation and a cell-cell adhesion molecule and cell-ECM molecule for metastasis.

Upregulation of cathepsin and MMPs in breast cancer patients who had menarche at less than 14 years of age may be because breast cancer in this group: 1) is hormone receptor negative; and 2) at this age the breast cells are extremely sensitive, thus pathogenesis of the breast leads to overexpression of cathepsin and MMP. ${ }^{16}$ So tumor 
cells and the surrounding stromal cells express a high level of these enzymes for tumor metastases.

To the best of our knowledge, there is no literature that links cathepsin and MMPs with breast cancer looking at parity as confounding variable. Our result does not show a significant difference between nulliparous and multigravida breast cancer patients. However, there is a significantly increased expression of cathepsin and MMPs in breast cancer tissue compared to normal breast tissue in women who give birth for the first time below 25 years of age,contrary to women who give birth for the first time at the age of 25 and above, this may be because the sample is small.

In these breast cancer patients, tumor MMP-2 were 4 times increased and MMP-9 activities were 7 times higher than normal tissue (Figure 4, $\mathrm{n}=36, \mathrm{P}<0.001$ ); however, CTSK enzymatic activity was 3 times higher than the enzymatic activity in non-tumor tissue $(\mathrm{n}=36, \mathrm{p}<$ 0.001), CTSL was 4 times higher (Figure 7, $\mathrm{n}=36, \mathrm{p}<$ $0.001)$. This may be because of increased activity of cathepsin and MMPs in the tumor micro-environment degrade the ECM. Our result is in agreement with Platt's findings. ${ }^{18}$

The increased level of these proteolytic enzymes in tumor cells may be because these enzymes are secreted not only by tumor cells but also by the stromal cells of breast tissue. Cancer cells extract energy through glycolysis, even though there is enough oxygen, which create acidic tumor microenvironment that initiate downstream signaling which leads to expression of CTSK and CTSL. Other factors proposed are over expression of ErbB2, auto activation of CTS-K, truncated nucleo-cytosolic translocation of CTSL. Cathepsin and MMPs proteolytically degrade the ECM to facilitate tumor penetration, invasion and metastasis. ${ }^{5}$

From the two cathepsins' research result, CTSL enzymatic activity was chiefly distinctive in breast tissue and it was significantly high in cancerous tissue compared to normal tissue. This is in contrast to the study by Platt, which showed that CTSK was significantly higher at all stages. ${ }^{16}$

Another family of proteases are MMPs which rely on metal ion and involved in tumor growth and metastasis. MMP-2 and MMP-9 are the two most studied protease family members and gelatin zymography recognizes and identifies their enzymatic activity, ${ }^{9}$ which is similar to the current study.
MMP-9 damages collagen IV and deteriorates the basement membrane and is known to play a significant role in the environment of tumor development and metastases. ${ }^{10}$ Fragments of collagen IV which are the degradation products of ECM, can act as a signaling molecules which control cell movement. ${ }^{20,21}$

Malignant breast tumors have increased MMP-9 enzymatic activity compared to normal breast tissue and this shows an increasing releasing and activation of MMP-9 in advanced stages of breast cancer. However, MMP-9 expression has also been taken as a positive prognostic marker in node-negative breast cancer. $^{22}$ In another research, advanced MMP-9 expression in cancer cells was related with fewer lympho-vascular penetration and lesser tumor grade. Hence, expression of MMP-9 is associated with inhibition and stimulation of cancer development and advancement, maybe all of these studies did not measure the actual MMP-9 activity. ${ }^{23}$

Zymographic study done using serum sample for control group by Scorilas et al $(2001)^{24}$ showed that the mean levels of enzymatic activities of circulating MMP-2 and MMP-9 in breast cancer patients were significantly higher than control serum $(\mathrm{P}<0.001)$. Besides this, it suggest that tissue samples tested for MMP-2 and MMP-9 activity may have remindful diagnostic value for differentiating subgroups of breast cancer patients. ${ }^{22}$

This study revealed the relationship between the tumor size and enzymatic activity. Cathepsin and MMPs are significantly high in small size tumors rather than in larger size tumors. This may be because a small tumor can easily detach from its primary site compared to larger tumor, which is fixed at the primary site. ${ }^{25}$ Therefore, a small tumor expresses these proteolytic enzymes from tumor cells as well as the surrounding stromal cells. Plus through upregulation of integrin $\alpha 3 \beta 1,{ }^{26}$ cancer tissue and hypoxia as a result of fast growth in small tissue, promote downstream signaling leading to increased expression of cathepsin and MMPs.

In this study it is observed that upregulation of cathepsin and MMPs in late stage breast cancer is contrary to Platt's study. ${ }^{18}$ This may be because late stage breast cancer is engaged in local as well as distant metastases. Therefore, these enzymes are highly expressed proteolytic enzymes in later stages to degrade cell-ECM adhesion molecules and cell-cell adhesion molecules that ease the tumor metastasis.

As of now, the most grounded indicators of breast cancer metastasis are lymph node involvement and histological grading. These test parameters are insufficient to distinguish 
the putative subgroup of patients within the same clinical category. It is much reported that breast cancer patients at the same stage and grade of disease can have various result outcomes and treatment responses. Thus, looking for new molecular biomarkers is essential and hence, it is an open area for various researchers. Specifically, the cysteine cathepsin and MMP enzyme activities have taken the attention of research investigators, because of their likely use as molecular biomarkers and treatment targets. Most of MMPs are released as dormant proenzyme forms and are exposed to controlled activation/inactivation at the cell-matrix border. Body fluids also have secreted proenzyme forms where they can be detected easily.

\section{Conclusion}

Breast cancer in Ethiopia predominantly peaks in the age cluster of 30-39 years. Study participants noticed the lump accidentally, and are diagnosed at a late stage. There are several reasons for a late diagnosis: the absence of a doctor's consultation for a mammogram; because it is considered expensive; there is no belief that a mammogram is necessary based on the symptoms; there is a problem with access to a mammography facility; it is a shame; and a curse of God.

The present study revealed that cathepsin and MMPs are upregulated in tumor tissue when compared to adjacent noncancerous normal tissues. These enzyme shows exaggerated proteolytic activities in the tumor microenvironment above their normal physiological proteolytic function.

The result shows there is upregulation of cathepsin and MMPs in multigravida and women who had their first live birth at a young age; there is no clear explanation for this unless the sample is small and non-representative. If there is a known mechanism for their upregulation, these enzymes could be used as prognostic indicators. Increased functional activity of these enzymes were captured in breast cancer patients who had early menarche.

Cathepsin and MMPs' upregulation weakens e-cadherin resulting in the weakening of cell-cell adhesion, truncated integrin result in weakening of cell-ECM interactions, also initiate downstream cancer survival signaling, that ultimately leads to tumor invasion and metastasis.

Cathepsin and MMPs are significantly higher in breast cancer patients with small tumor size and late stage of breast cancer. Thus, understanding and investigating cathepsin and MMPs' intervened tissue alteration and remodeling is significant for fundamental research science and for clinical purposes, such as biomarkers.

\section{Abbreviation}

BCA, bicinchoninic acid assay; CTSK, cathepsin K; CTSL, cathepsin L; ELISA, enzyme linked immunosorbent assay; DNA, deoxy ribonucleic acid; FDA, Federal Drug Association; MMPs, matrix metalloproteinase; PAGE, polyacrylamide gel electrophoresis; SDS-PAGE, sodium dodecyl sulphate - polyacrylamide gel electrophoresis; SDS, sodium dodecyl sulphate; SNP, single nucleotide polymorphism; WHO, World Health Organization.

\section{Data Sharing Statement}

All information collected or analyzed during this study is included in this article.

\section{Acknowledgments}

The authors would like to recognize, the study participants for their collaboration and readiness. In addition, we would like to extend our sincere gratitude to the Addis Ababa University of Biochemistry Department for their administrative and financial support. ${ }^{27}$

\section{Author Contributions}

All authors made a significant contribution to the work reported, whether that is in the conception, study design, execution, acquisition of data, analysis and interpretation, or in all these areas; took part in drafting, revising or critically reviewing the article; gave final approval of the version to be published; have agreed on the journal to which the article has been submitted; and agree to be accountable for all aspects of the work.

\section{Funding}

The authors are grateful to Addis Ababa University, College of Health Sciences School of Medicine for their financial support.

\section{Disclosure}

The authors state that there is no conflict of interest in this work.

\section{References}

1. Mohamed M, Menon M, Nebeyou S, Mahteme B, Wondimagegn T, Daniel S. Blood and tissue enzymatic activities of GDH and LDH, index of glutathione, and oxidative stress among breast cancer patients attending Referral Hospitals of Addis Ababa, Ethiopia: hospital-based comparative cross-sectional study. Oxid Med Cell Longev. 2018;2018:12.

2. Ferlay J, Soerjomataram I, Dikshit R, et al. Cancer incidence and mortality worldwide: sources, methods and major patterns in GLOBOCAN 2012. Int $J$ Cancer. 2015;136(5):E359-86. doi:10.1002/ijc. 29210 
3. Anderson BO, Shyyan R, Eniu A, et al. Breast Cancer in LimitedResource Countries: an Overview of the Breast Health Global Initiative 2005 Guidelines. Breast J. 2006;12(s1):S3-S15 doi:10.1111/j.1075-122X.2006.00199.x

4. Tfayli A, Temraz S, Abou Mrad R, Shamseddine A. Breast cancer in low-and middle-income countries: an emerging and challenging epidemic. J Oncol. 2010;2010. doi:10.1155/2010/490631

5. Bartsch JE, Staren ED, Appert HE. Matrix metalloproteinase expression in breast cancer. J Surg Res. 2003;110(2):383-392. doi:10.1016/ S0022-4804(03)00007-6

6. Mohamed MM, Sloane BF. Cysteine cathepsins: multifunctional enzymes in cancer. Nat Rev Cancer. 2006b;6(10). doi:10.1038/nrc1949

7. Dumas JE, Platt MO. Systematic optimization of multiplex zymography protocol to detect active cathepsins $\mathrm{K}, \mathrm{L}, \mathrm{S}$, and $\mathrm{V}$ in healthy and diseased tissue: compromise among limits of detection, reduced time, and resources. Mol Biotechnol. 2013;54(3):1038-1047.

8. Barry ZT, Platt MO. Cathepsin S cannibalism of cathepsin K as a mechanism to reduce type I collagen degradation. $J$ Biol Chem. 2012;287(33):27723-27730. doi:10.1074/jbc.M111.332684

9. Das S, Banerji A, Frei E, Chatterjee A. Rapid expression and activation of MMP-2 and MMP-9 upon exposure of human breast cancer cells (MCF-7) to fibronectin in serum free medium. Life Sci. 2008;82 (9):467-476. doi:10.1016/j.lfs.2007.12.013

10. Duffy M, McCarthy K. Matrix metalloproteinases in cancer: prognostic markers and targets for therapy (review).. Int J Oncol. 1998;12 (6):1343-1348. doi:10.3892/ijo.12.6.1343

11. Hirvonen R, Talvensaari-Mattila A, Paakko P, TurpeenniemiHujanen T. Matrix Metalloproteinase-2 (MMP-2) in T1-2 N0 breast carcinoma. Breast Cancer Res Treat. 2003;77(1):7.

12. La Rocca G, Pucci-Minafra I, Marrazzo A, Taormina P, Minafra S. Zymographic detection and clinical correlations of MMP-2 and MMP-9 in breast cancer sera. Br J Cancer. 2004;90(7):1414-1421. doi: $10.1038 /$ sj.bjc. 6601725

13. Kalli S, Semine A, Cohen S, Naber SP, Makim SS, Bahl M. American joint committee on cancer's staging system for breast cancer: what the radiologist needs to know. Radiographics. 2018;38 (7):1921-1933. doi:10.1148/rg.2018180056

14. Amin MB, Greene FL, Edge SB, et al. The eighth edition AJCC cancer staging manual: continuing to build a bridge from a population-based to a more "personalized" approach to cancer staging. CA Cancer J Clin. 2017;67(2):93-99. doi:10.3322/caac.21388

15. Smith KM, Xu Y. Tissue sample preparation in bioanalytical assays. Bioanalysis. 2012;4(6):741-749. doi:10.4155/bio.12.19

16. Chen B, Platt MO. Multiplex zymography captures stage-specific activity profiles of cathepsins $\mathrm{K}, \mathrm{L}$, and $\mathrm{S}$ in human breast, lung, and cervical cancer. $J$ Transl Med. 2011;9(1):109. doi:10.1186/14795876-9-109
17. Akin-Odanye E, Asuzu CC, Popoola OA. Measured effect of some socio-demographic factors on depression among breast cancer patients receiving chemotherapy in Lagos State University Teaching Hospital (LASUTH). Afr Health Sci. 2011;11:3.

18. Dumas JE, Platt MO. Systematic optimization of multiplex zymography protocol to detect active cathepsins $\mathrm{K}, \mathrm{L}, \mathrm{S}$, and $\mathrm{V}$ in healthy and diseased tissue: compromise among limits of detection, reduced time, and resources. Mol Biotechnol. 2013;54(3):1038-1047.

19. Miller BA, Hankey BF, Thomas TL. Impact of sociodemographic factors, hormone receptor status, and tumor grade on ethnic differences in tumor stage and size for breast cancer in US women. $A m$ J Epidemiol. 2002;155(6):534-545. doi:10.1093/aje/155.6.534

20. Jinga D, Blidaru A, Condrea I, et al. MMP-9 and MMP-2 gelatinases and TIMP-1 and TIMP-2 inhibitors in breast cancer: correlations with prognostic factors. $J$ Cell Mol Med. 2006;10(2):499-510. doi:10.1111/j.1582-4934.2006.tb00415.x

21. Quaranta M, Daniele A, Coviello M, et al. MMP-2, MMP-9, VEGF and CA 15.3 in breast cancer. Anticancer Res. 2007;27 (5B):3593-3600.

22. Scorilas A, Karameris A, Arnogiannaki N, et al. Overexpression of matrix-metalloproteinase-9 in human breast cancer: a potential favourable indicator in node-negativeatients. Br J Cancer. 2001;84 (11): 1488 .

23. Reich R, Supuran CT, Breuer E. Tumor microenvironment as target in cancer therapy. Annual Rep Med Chem. 2014;49:269-284.

24. Scorilas A, Karameris A, Arnogiannaki N, et al. Overexpression of matrix-metalloproteinase-9 in human breast cancer: a potential favourable indicator in node-negative patients. $\mathrm{Br} J$ Cancer. 2001;84(11):1488-1496. doi:10.1054/bjoc.2001.1810

25. Pellikainen JM, Ropponen KM, Kataja VV, Kellokoski JK, Eskelinen MJ, Kosma V-M. Expression of matrix metalloproteinase (MMP)-2 and MMP-9 in breast cancer with a special reference to activator protein-2, HER2, and prognosis. Clin Cancer Res. 2004;10 (22):7621-7628. doi:10.1158/1078-0432.CCR-04-1061

26. Têtu B, Brisson J, Wang CS, et al. The influence of MMP-14, TIMP-2 and MMP-2 expression on breast cancer prognosis. Breast Cancer Res. 2006;8(3):R28. doi:10.1186/bcr1503

27. Solomon $\mathrm{T}$ Zymographic detection and clinical correlations of cysteine cathepsins and Matrix Metalloproteases in Human Breast Cancer Tissue: AAU; 2016. Available from: http:/etd.aau.edu.et/han dle/1234567892995. Accessed 19 January 2020

\section{Publish your work in this journal}

Breast Cancer - Targets and Therapy is an international, peer-reviewed open access journal focusing on breast cancer research, identification of therapeutic targets and the optimal use of preventative and integrated treatment interventions to achieve improved outcomes, enhanced survival and quality of life for the cancer patient.
The manuscript management system is completely online and includes a very quick and fair peer-review system, which is all easy to use. Visit http://www.dovepress.com/testimonials.php to read real quotes from published authors. 